\title{
A little stress is good: IFN- $\gamma$, demyelination, and multiple sclerosis
}

\author{
Jason R. Lees ${ }^{1}$ and Anne H. Cross ${ }^{2,3}$
}

\author{
${ }^{1}$ Department of Molecular Biology and Pharmacology, ${ }^{2}$ Department of Neurology and Neurosurgery, and \\ ${ }^{3}$ Hope Center for Neurological Disorders, Washington University School of Medicine, St. Louis, Missouri, USA.
}

\begin{abstract}
The exact role(s) of the cytokine IFN- $\gamma$ in the demyelinating disease multiple sclerosis remain controversial, with evidence suggesting both detrimental and protective effects of the cytokine in MS and MS models such as EAE. The study by Lin and coworkers in this issue of the JCI produces evidence that protective effects of IFN- $\gamma$ on mature oligodendrocytes during EAE induction are mediated via activation of the pancreatic ER kinase (PERK), resulting in induction of the endoplasmic reticular stress response pathway (see the related article beginning on page 448). Modulation of this stress pathway has what we believe to be novel therapeutic potential for MS.
\end{abstract}

MS is a debilitating chronic disease of the CNS that affects over 400,000 people in the United States. MS is marked by infiltration of the CNS by inflammatory cells and by destruction of oligodendrocytes (ODCs) and the associated myelin sheaths within CNS white matter (1). The amount of ODC loss in MS is variable and appears to evolve into 2 major patterns in active lesions (2). One type is characterized by extensive ODC destruction. Although oligodendroglial precursor cells are often present, these cells are not associated with significant remyelination. Preservation of ODCs, allowing the possibility of remyelination, would be highly beneficial to these patients. In contrast, the second lesion type is characterized by ODC survival and ODC progenitor recruitment, often with significant remyelination (2).

IFN- $\gamma$ is a major cytokine found in MS lesions, and its levels are greatly increased during MS activity. Inflammation in both MS and EAE is associated with an IFN- $\gamma-$ producing $T h 1$ response $(3,4)$. In rodent MS models, skewing of the cytokine milieu away from a Th1 lineage and toward a Th2producing response clearly ameliorates EAE $(5,6)$. Administration of IFN- $\gamma$ to people with MS aggravates clinical disease, resulting in increased numbers of relapses in patients (7). However, several studies

Nonstandard abbreviations used: $\operatorname{eIF} 2 \alpha, \alpha$ subunit of the eukaryotic translation initiation factor 2; ODC, oligodendrocyte; PERK, pancreatic endoplasmic reticulum kinase.

Conflict of interest: The authors have declared that no conflict of interest exists.

Citation for this article: J. Clin. Invest. 117:297-299 (2007). doi:10.1172/JCI31254. have suggested that IFN- $\gamma$ may actually have a beneficial role in EAE and other demyelinating animal models. Induction of EAE in mice lacking IFN- $\gamma$ or the IFN- $\gamma$ receptor as well as administration of antiIFN- $\gamma$ antibodies all have resulted in more severe disease, suggesting that IFN- $\gamma$ may limit disease (8-10). Results from studies using the ODC toxin cuprizone indicated that IFN- $\gamma$ treatment of ODCs might render them less susceptible to the toxin (11).

Because of the prominent role of IFN- $\gamma$ in the development of an autoimmune response, several immunomodulatory functions of IFN- $\gamma$ have been suggested as potential disease-altering mechanisms. These mechanisms range from affecting the initiation of the $\mathrm{T}$ cell anti-CNS response, to induction of apoptosis in CNS-infiltrating leukocytes, to repression of the induction of IL-17-producing encephalitogenic $\mathrm{T}$ cells $(8-10,12)$.

\section{Enhancing ODC survival}

In this issue of the JCI, Lin, Popko, and colleagues have provided compelling evidence of an unexpected mechanism by which IFN- $\gamma$ could prove beneficial: a direct protective effect on mature ODC survival during EAE in mice (13). Using CNS-specific IFN- $\gamma$ production regulated by tetracycline-repressed control of a glial fibrillary acidic protein (GFAP) promoter, the authors examined the effect of early IFN- $\gamma$ production confined to the CNS on subsequent EAE. By means of this temporally restricted expression of IFN- $\gamma$ production by astrocytes, the effects of CNS-specific IFN- $\gamma$ production on $\mathrm{EAE}$ induction in an otherwise developmen- tally normal system were examined. ODC survival was significantly enhanced upon IFN- $\gamma$ expression in the CNS following EAE induction. The survival of ODCs was associated with a reduced disease course and less demyelination. No association of this protective effect and the effects of IFN- $\gamma$ on the immune system was apparent. Indeed, the production of CNS IFN- $\gamma$ had surprisingly little effect on microglia and invading macrophages at any time reported, nor did it involve regulation of initial immune cell entry or cytokine production (13).

\section{A role for the ER}

Several groups have previously suggested a role for ER stress-induced cell death in the initiation of autoimmune and neurodegenerative diseases $(14,15)$. Induction of ER stress has also been shown to result in reduction of protein synthesis and concomitant protection of cells from future stress $(16,17)$. This cytoprotective pathway has been called the integrated stress response and relies on activation of the pancreatic ER kinase (PERK). As IFN- $\gamma$ is known to induce PERK activation, Lin et al. (13) assessed the capacity of CNS-specific delivery of IFN- $\gamma$ to induce the integrated stress response. Induction of CNS-specific IFN- $\gamma$ resulted in activation of the integrated stress response initiated by PERK activation followed by phosphorylation of the $\alpha$ subunit of the eukaryotic translation initiation factor $2(\mathrm{eIF} 2 \alpha)$. Support for a critical role for PERK induction of the integrated stress response in ODC protection was found when PERK deficiency abrogated the protective effects of IFN- $\gamma$.

The present findings by Lin et al. (13) are in contrast to previous reports that the ER stress response induces apoptosis in newly developed ODCs responsible for remyelination following demyelination (18). This same group of investigators has previously shown that IFN- $\gamma$ induced ER stress in myelinating ODCs during development or remyelination, which led to ODC death and myelin abnormalities, while PERK 


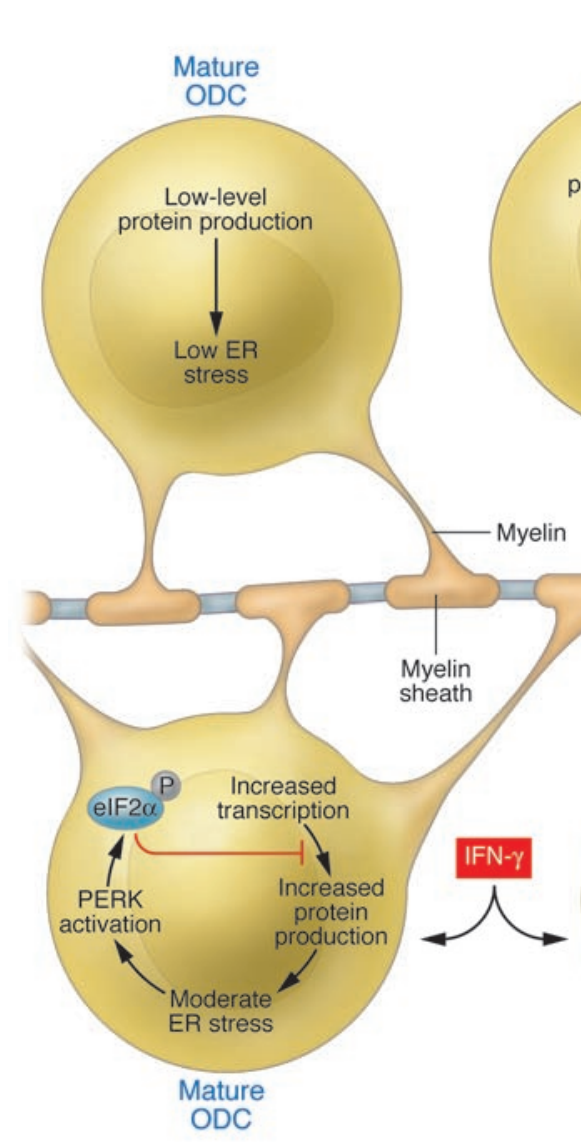

activation provided some small degree of protection from these effects $(18,19)$. The increased IFN- $\gamma$-induced death of remyelinating ODCs in PERK-deficient mice observed in the earlier studies was reiterated in the current paper (13) by the apparent increase in cellular damage associated with IFN- $\gamma$ treatment of PERK-deficient mice (see Figure 2, A and C, in ref. 13) as well as the slightly higher death rate even in the absence of CNS-specific IFN- $\gamma$ (see Table 1 in ref. 13).

\section{Understanding the conflicting actions of IFN- $\gamma$ in demyelination}

How does one resolve these conflicting reports? Lin et al. (13) speculate that the differences observed relate to the timing of IFN- $\gamma$ administration and to the differential effects of ER stress induced by IFN- $\gamma$ on actively myelinating ODCs versus mature ODCs, suggesting a model in which the response to IFN- $\gamma$ by a given cell would be directly related to its relative rate of protein production. IFN- $\gamma$-induced protein expression (of molecules such as MHC class I) would represent the final push necessary to convert a protective, modest level of ER stress response in remyelinating ODCs actively involved in high-level protein production to a destructive, severe ER stress response. In contrast, protein expression induced by IFN- $\gamma$ would only be sufficient to drive mature ODCs, with their lower baseline protein production, to a modest level of ER stress, with a concomitant PERK-induced protective stress response. Thus, in mature ODCs functioning only to maintain myelin made at a prior time, the integrated stress response would be protective. In contrast, actively remyelinating ODCs would have increased sensitivity to the additional ER stress associated with IFN- $\boldsymbol{\gamma}$-induced protein expression resulting in ODC cell destruction (Figure 1).

The possibility that the IFN- $\gamma$ response is different for different stages of ODC differentiation fits well with many of Popko and colleagues' previous findings regarding the involvement of PERK $(18,19)$. Further dissection of the paradigm reported here (13) would also be interesting with regard to the role of ODC death in the recruitment of $T$ cells in the EAE model of disease and its implications for human MS. This paradigm may provide an opportunity to differentiate

\section{Figure 1}

A model of the differential responses to IFN- $\gamma$ of ODCs during their differentiation. The schematic shows the response of ODCs, at 2 different stages of development, to IFN- $\gamma$ delivered to the CNS before EAE onset in mice. Low constitutive protein production allows mature ODCs (top left) to survive the increase in ER stress that is associated with the initial interaction with IFN- $\gamma$ (bottom left). Having survived the original stressor, mature ODCs induce activation of PERK. PERK activation in turn induces elF2 $\alpha$ phosphorylation, ultimately allowing ODCs to acquire resistance to further ER stress. The acquired resistance to ER stress protects cells from apoptotic mechanisms associated with EAE, allowing the cells to maintain functional myelin sheaths and concomitantly reducing clinical symptoms observed in MS. In contrast, a high level of constitutive protein production in developing ODCs (top right) results in toxic levels of ER stress following IFN- $\gamma$ treatment (bottom right), resulting in an increased risk of apoptosis in developing ODCs. what aspects of pathophysiology are directly caused by ODC death and what aspects are regulated by inflammatory mechanisms independent of demyelination.

Together, the data discussed here indicate that the regulation of the balance of cell stress is likely to play a vital role in the response of ODCs to the highly complex pathologic mechanisms involved in demyelinating disease. To compound the complexity of the situation, the role of ODC death in human MS may vary from patient to patient. Some patients may have a type of MS in which ODC precursors are critical for remyelination. Based upon these animal models, IFN- $\gamma$ would be detrimental in that situation. On the other hand, those people with MS characterized predominantly by ODC death might benefit from IFN- $\gamma$ delivered to the CNS at the proper time. CNS-specific IFN- $\gamma$ expression has been variably reported as increased, decreased, or unchanged in MS patients. It would be of considerable interest to correlate the level of CNS-specific IFN- $\gamma$ and the degree of IFN- $\gamma$-induced integrated stress response with the predominant pathology of ODCs in individuals affected with MS. Indeed, 
an understanding of these relationships would be required before attempting to alter ODC stress responses in MS patients for therapeutic benefit.

\section{Acknowledgments}

We would like to acknowledge John H. Russell for his helpful review and discussion of this commentary.

Address correspondence to: Anne H. Cross, Department of Neurology and Neurosurgery, Washington University School of Medicine, 660 S. Euclid Avenue, Box 8111, St. Louis, Missouri 63110, USA. Phone: (314) 362-3293; Fax: (314) 747-1345; E-mail: crossa@neuro.wustl.edu.

\footnotetext{
1. Giovannoni, G., and Hartung, H.P. 1996. The immunopathogenesis of multiple sclerosis and Guillain-Barre syndrome. Curr. Opin. Neurol. 9:165-177.

2. Lucchinetti, C., et al. 1999. A quantitative analysis of oligodendrocytes in multiple sclerosis lesions. A study of 113 cases. Brain. 122:2279-2295.

3. Liblau, R.S., Singer, S.M., and McDevitt, H.O. 1995. Th1 and Th2 CD4+ T cells in the pathogenesis of organ-specific autoimmune diseases. Immunol.
}

Today. 16:34-38.

4. Zamvil, S.S., and Steinman, L. 1990. The T lymphocyte in experimental allergic encephalomyelitis. Annu. Rev. Immunol. 8:579-621.

5. Soos, J.M., et al. 2002. Cutting edge: oral type I IFN-tau promotes a Th2 bias and enhances suppression of autoimmune encephalomyelitis by oral glatiramer acetate. J. Immunol. 169:2231-2235.

6. Youssef, S., et al. 2002. The HMG-CoA reductase inhibitor, atorvastatin, promotes a Th2 bias and reverses paralysis in central nervous system autoimmune disease. Nature. 420:78-84

7. Panitch, H.S., Hirsch, R.L., Schindler, J., and Johnson, K.P. 1987. Treatment of multiple sclerosis with gamma interferon: exacerbations associated with activation of the immune system. Neurology. 37:1097-1102.

8. Furlan, R., et al. 2001. Intrathecal delivery of IFNgamma protects C57BL/6 mice from chronicprogressive experimental autoimmune encephalomyelitis by increasing apoptosis of central nervous system-infiltrating lymphocytes. J. Immunol. 167:1821-1829.

9. Willenborg, D.O., Fordham, S., Bernard, C.C., Cowden, W.B., and Ramshaw, I.A. 1996. IFNgamma plays a critical down-regulatory role in the induction and effector phase of myelin oligodendrocyte glycoprotein-induced autoimmune encephalomyelitis. J. Immunol. 157:3223-3227.

10. Willenborg, D.O., Fordham, S.A., Staykova, M.A., Ramshaw, I.A., and Cowden, W.B. 1999. IFNgamma is critical to the control of murine autoimmune encephalomyelitis and regulates both in the periphery and in the target tissue: a possible role for nitric oxide. J. Immunol. 163:5278-5286.

11. Gao, X., et al. 2000. Interferon-gamma protects against cuprizone-induced demyelination. Mol. Cell. Neurosci. 16:338-349.

12. Komiyama, Y., et al. 2006. IL-17 plays an important role in the development of experimental autoimmune encephalomyelitis. J. Immunol. 177:566-573.

13. Lin, W., et al. 2007. The integrated stress response prevents demyelination by protecting oligodendrocytes against immune-mediated damage. J. Clin. Invest. 117:448-456. doi:10.1172/JCI29571.

14. Rao, R.V., Ellerby, H.M., and Bredesen, D.E. 2004. Coupling endoplasmic reticulum stress to the cell death program. Cell Death Differ. 11:372-380.

15. Oyadomari, S., Araki, E., and Mori, M. 2002. Endoplasmic reticulum stress-mediated apoptosis in pancreatic beta-cells. Apoptosis. 7:335-345.

16. Harding, H.P., et al. 2003. An integrated stress response regulates amino acid metabolism and resistance to oxidative stress. Mol. Cell. 11:619-633.

17. Lu, P.D., et al. 2004. Cytoprotection by pre-emptive conditional phosphorylation of translation initiation factor 2. EMBO J. 23:169-179.

18. Lin, W., et al. 2006. Interferon-gamma inhibits central nervous system remyelination through a process modulated by endoplasmic reticulum stress. Brain. 129:1306-1318.

19. Lin, W., Harding, H.P., Ron, D., and Popko, B. 2005. Endoplasmic reticulum stress modulates the response of myelinating oligodendrocytes to the immune cytokine interferon-gamma. J. Cell Biol. 169:603-612

\title{
Notch: a mastermind of vascular morphogenesis
}

\author{
Leonard M. Anderson and Gary H. Gibbons
}

Cardiovascular Research Institute, Morehouse School of Medicine, Atlanta, Georgia, USA.

\begin{abstract}
The way in which multiple cell types organize themselves into a carefully sculpted, 3D labyrinth of vessels that regulate blood flow throughout the body has been a longstanding mystery. Clinicians familiar with congenital cardiovascular disease recognize how genetic variants and modest perturbations in this complex set of spatiotemporal interactions and stochastic processes can result in life-threatening anomalies. Although the mystery is not yet fully solved, we are poised at an exciting juncture, as insights from murine disease models are converging with advances in human genetics to shed new light on puzzling clinical phenotypes of vascular disease. The study by High et al. in this issue of the JCI establishes a model system that mimics clinical features of congenital cardiovascular disease and further defines the role of the Notch signaling pathway in the neural crest as an essential determinant of cardiovascular structure (see the related article beginning on page 353).
\end{abstract}

\section{The process of vascular morphogenesis}

In the embryo, endothelial precursors initially assemble into a primitive plexus of channels that expand by sprouting and

Nonstandard abbreviations used: HERP, HES-related repressor protein; HES, hairy and enhancer of split; MAML, mastermind-like; Tbx1, T-box 1.

Conflict of interest: The authors have declared that no conflict of interest exists.

Citation for this article: J. Clin. Invest. 117:299-302 (2007). doi:10.1172/JCI31288. remodeling into a highly organized arborization of vessels that ramify throughout the body. Studies in various knockout mouse models suggest that VEGF, angiopoietins, and PDGF provide local cues that promote vascular morphogenesis and the investment of the endothelial channels with a VSMC layer. The architecture of the vascular tree is further refined to very precise dimensions in accordance with biomechanical parameters of shear and radial stresses $(1,2)$.
In a classic series of seminal studies involving quail-chick chimeras and tissue ablation experiments, Kirby and colleagues established that the cardiac neural crest plays an essential role in establishing the pattern of the vertebrate vascular system (3). The molecular mechanisms underlying these phenomena are gradually being elucidated (Table 1). The cardiac neural crest (an ectodermal cell population arising from the dorsal neural tube) migrates to populate the aortic arch arteries and cardiac outflow tract. The migrating cardiac neural crest cells contribute to the septation of the truncus arteriosus into a separate pulmonary artery and aorta. Similarly, a subpopulation of these neural crest cells becomes part of the mass of VSMCs that contribute to the formation of the pulmonary trunk, ductus arteriosus, carotid arteries, and proximal subclavian arteries (3-5). The study reported by High et al. in this issue of the JCI (6) provides the first demonstration to our knowledge that the Notch transcriptional cascade within the neural crest plays an essential mediator role in VSMC differ- 